\title{
Chronic Organic Arsenic Induced Liver Ultra Structural Damage
}

Shahida Saharudin ${ }^{1}$, Norlelawati A. Talib², Nor Zamzila Abdullah², Jamalludin Ab. Rahman $^{3}$, Zunariah Buyong ${ }^{1}$

${ }^{1}$ Department of Basic Medical Science, Kulliyyah of Medicine, International Islamic University Malaysia

${ }^{2}$ Department of Pathology and Laboratory Medicine, Kulliyyah of Medicine, International Islamic University Malaysia

${ }^{3}$ Department of Community Medicine, Kulliyyah of Medicine, International Islamic University Malaysia

Presenter: Shahida Saharudin

Introduction: Inorganic arsenic is one of the environmental toxins that has been associated with increased risk of cardiovascular diseases and vascular contributions to liver diseases. It has generally been thought to be more toxic than organic arsenic. In human liver, inorganic arsenic promotes vascular remodelling, portal fibrosis and hypertension. The purpose of the current study was to determine whether chronic exposure to organic arsenic impair liver ultrastructure as in inorganic arsenic exposure. Materials and Methods: Twenty eight male SpragueDawley rats were divided into 2 groups with their own control group. They received oral intubation of monosodium- methylarsonate (MSMA) at $63.30 \mathrm{mg} / \mathrm{kg}$ body weight for 4 and 6 months duration respectively while the control groups received distilled water. The liver of euthanized rats were perfused- fixed with glutaraldehyde for transmission electron microscopy processing. Results: TEM revealed a marked reduction in the number of mitochondria in both treatment groups. Some typical features of apoptosis are present with pyknotic nuclei of hepatocytes and disintegrated hepatic cytoplasm in 4- month groups. The microvilli of hepatocyte are almost completely absent with the presence of many fibre bundles (collagen fibres) widening the space of Disse. In 6-month treatment group, disintegrated cytoplasms are more prominent with degrading mitochondria of varying stages. Liver sinusoidal endothelial cells (LSEC) of 6-month treatment group are noted to have chromatin condensation with few caveolae seen. Conclusion: Chronic exposure of MSMA leads to necrotic changes of hepatocytes and apoptotic changes in LSEC and collagenisation in the space of Disse. 